\title{
Hardware Acceleration of Sparse Support Vector Machines for Edge Computing
}

\author{
Vuk Vranjkovic*, Rastislav Struharik \\ Department of Power, Electronic and Telecommunication Engineering, Faculty of Technical Sciences, \\ University of Novi Sad, \\ Trg Dositeja Obradovica 6, 21000 Novi Sad, Serbia \\ bykbpa@uns.ac.rs
}

\begin{abstract}
In this paper, a hardware accelerator for sparse support vector machines (SVM) is proposed. We believe that the proposed accelerator is the first accelerator of this kind. The accelerator is designed for use in field programmable gate arrays (FPGA) systems. Additionally, a novel algorithm for the pruning of SVM models is developed. The pruned SVM model has a smaller memory footprint and can be processed faster compared to dense SVM models. In the systems with memory throughput, compute or power constraints, such as edge computing, this can be a big advantage. The experiments on several standard datasets are conducted, which aim is to compare the efficiency of the proposed architecture and the developed algorithm to the existing solutions. The results of the experiments reveal that the proposed hardware architecture and SVM pruning algorithm has superior characteristics in comparison to the previous work in the field. A memory reduction from $3 \%$ to $85 \%$ is achieved, with a speed-up in a range from 1.17 to 7.92.
\end{abstract}

Index Terms-Support vector machines; Hardware accelerator architectures; Edge computing.

\section{INTRODUCTION}

Support Vector Machine (SVM) is one kind of machine learning algorithms, firstly introduced in [1]. SVMs were one of the most popular predicting models until Convolutional Neural Networks (CNN) have been proposed. Also, there is work on hybrid models of CNN and SVM, e.g., in [2], [3].

As with other supervised machine learning algorithms, SVM contains two phases: a learning phase and a predicting phase. The SVM model approximates unknown function U. In the learning phase, input to the SVM training algorithm is a training set with $m$ instances, each of which has $n$ attributes

$$
T S=\left\{x_{i}, y_{i}\right\}, i=1 . . m, x_{i} \in X \subseteq R^{n}, y \in Y=\{+1,-1\}
$$

The output of the SVM learning phase is a linear

Manuscript received 24 February, 2020; accepted 29 April, 2020

This work was partially supported by Serbian Ministry of Education and Science under a grant (No. TR32016 - "Innovative electronic components and systems based on inorganic and organic technologies embedded in consumer goods and products"). This project has received funding from the European Union's Horizon 2020 research and innovation programme under a Grant Agreement (No. 856697). classification function $L$ in a form

$$
L: X \rightarrow Y, L(x)=w \times x+b, w \in R^{n}, b \in R .
$$

The function $L$ approximates the unknown function $: X \rightarrow$ $Y$

Function $L$, as previously defined, can correctly classify only the linearly separable training set. To be able to classify instances that belong to non-linearly separable classes, one can apply a non-linear mapping $\varphi$ to input space $X$ to transform the original input feature space to a highdimensional feature space $Z \subseteq R^{k}, k>n$. The same linear classification function can be used to separate points in this high-dimensional feature space, achieving non-linear classification in the original space $X$. Using the kernel trick, the SVM classifier still can work in the original space, so the non-linear classification function can be expressed in the form

$$
F(x)=w \times \phi(x)+b .
$$

SVM splits points in the input feature space with a linear hyperplane. The hyperplane is located at a maximal distance from the training set instances of each class that is closest to the hyperplane. In general, this cannot be done without errors for all training set instances, so the SVM algorithm allows some training set instances to be incorrectly classified. The problem of finding an optimal splitting hyperplane can be described formally as the constrained quadratic programming $(\mathrm{CQP})$ problem:

$$
\begin{gathered}
\min _{w, b}\left[\frac{1}{2}\|w\|^{2}+C \sum_{i=1}^{m} \varepsilon_{i}\right], \\
y_{i}(w \times \phi(x)+b) \geq 1-\varepsilon_{i}, C>0, \varepsilon_{i} \geq 0, \forall i \in 1 \ldots m .
\end{gathered}
$$

The first part of the minimization function puts the hyperplane at the maximal margin, while the second part changes the position of the hyperplane in a way that minimizes the number of training set instances that will be misclassified. These two criteria are contradictory, so the parameter $\mathrm{C}$ defines a trade-off between them.

Using a method of Lagrange multipliers, the original CQP problem can be transformed into its dual form, which is easier to solve (6)-(9): 


$$
\begin{gathered}
\min _{\alpha}\left[\frac{1}{2} \alpha^{T} W \alpha+r^{T} \alpha\right], \\
w_{i j}=y_{i} y_{j} K\left(x_{i}, x_{j}\right), \\
K\left(x_{i}, x_{j}\right)=\phi\left(x_{i}\right) \phi\left(x_{j}\right), \\
0 \leq \alpha_{i} \leq C, r_{i}= \pm 1, \forall i \in 1 \ldots m, y^{T} \alpha=0 .
\end{gathered}
$$

The $m \times m$ matrix $W$ is symmetric positive semidefinite, and its elements are $w_{i j}$. The function $K$ is called kernel and it implicitly defines a non-linear mapping - $\varphi$. There are several popular kernels in use, some of them are:

$$
\begin{gathered}
K\left(x_{i}, x_{j}\right)=\left(x_{i} \times x_{j}+1\right)^{d} \text { : polynomial }, \\
K\left(x_{i}, x_{j}\right)=e^{-\gamma\left\|x_{i}-x_{j}\right\|^{2}}: \text { radial basis function, } \\
K\left(x_{i}, x_{j}\right)=\tanh \left(a x_{i} \times x_{j}+b\right): \text { sigmoid } .
\end{gathered}
$$

The parameters of the kernel are $d, \gamma, a$, and $b$.

More details about the theory behind the SVM training can be found in [4].

After the training procedure completes, some of the Lagrange multipliers will be zero, and others will be nonzero. The training set instances corresponding to the nonzero multipliers from input data set are called support vectors. Let $l$ be the number of support vectors $(1 \leq m)$.

The SVM training algorithm outputs a non-linear approximation function in the following form

$$
V: X \rightarrow Y, V(x)=b+\sum_{i=1}^{l} y_{i} \alpha_{i} K\left(s_{i}, x\right),
$$

where $S_{i}$ is the support vector and $x$ is the input instance.

The evaluation of the function $V(x)$ for the unknown input instance is called classification. This is the second phase of the SVM algorithm. If the value of the function is greater than zero, the input instance is classified as a class with label +1 , otherwise, it is labeled as a class -1 .

The SVM algorithm can be generalized to do a multiclass classification also. One approach for this generalization is given in [5].

The efficient algorithm for the training of SVM is a Sequential Minimal Optimization (SMO), firstly introduced in [6]. Several additional optimizations of the SMO algorithm have been proposed, one example being [7].

Machine learning algorithms are being used in different kinds of applications, ranging from servers to embedded systems. Deploying machine learning models to embedded systems is especially difficult because of the size of the models. Therefore, the compression and reduction of the size of the models attracted a lot of research effort [8]. The compression of the size of SVM models is presented in [12]. The main idea in those papers for the reduction of SVM size is in a smart selection of support vectors during the training of SVMs. In this paper, we present a complementary idea for the size reduction of SVMs: the removal of attributes from support vectors. To the best of our knowledge, this approach to the reduction of SVM size has not been previously reported for SVMs.
We propose the AST-SVM algorithm for training of attribute sparse SVMs, and the ASA-SVM hardware accelerator that can take advantage of this kind of sparse SVMs. The presented accelerator is aimed for use in "FPGA" (Field-Programmable Gate Array) systems, and it is especially useful for embedded and edge applications, where designers are constrained with severe computational, memory throughput, and power limitations. The AST-SVM algorithm produces SVMs with an increased number of zeroes in model parameters. By operating on these sparse support vector representations, the ASA-SVM accelerator usually requires less memory for the storage of support vectors, and by skipping calculations of zero products during classification, it can reach higher performance than comparable dense accelerators.

The hardware acceleration of SVMs has been an interesting topic in the research community, resulting in several proposed hardware architectures [14]]-[[19]. In contrast to the ASA-SVM, all previously proposed architectures operate on dense SVMs. As far as we know, the ASA-SVM accelerator is the first to operate on sparse SVMs.

The rest of this paper is structured as follows. The algorithm for pruning SVMs is given in section II. The AST-SVM algorithm sets a predefined number of attributes of training instances to zero; therefore, reducing the size of the classifier after the training is done. Section III contains a description of the ASA-SVM architecture, which is designed to take advantage of sparse SVMs, which contains a large number of zeroes in each of the support vectors. In that way, a higher processing performance can be achieved, as well as less memory usage. In section IV, the experimental results of the AST-SVM algorithm and ASASVM architecture are presented for various standard datasets. Section V contains conclusions.

\section{AtTribute SpARSE TRAINING SVM (AST-SVM) ALGORITHM}

The AST-SVM algorithm uses a simple method for choosing which values to eliminate. Although simple, the method gives good results. The presented algorithm uses standard SVM training as the sub-task. In the AST-SVM algorithm, some of the values of attributes from some of the input instances of the input training set are set to zero. Then, the standard SVM training algorithm is called. After the SVM training is completed, the output is the sparse SVM model.

The algorithm starts with some training dataset. At every iteration, the algorithm keeps all training instances sorted by their number of non-zero values. The training instance with the biggest number of non-zero values is chosen for attribute elimination. Then, the attribute whose absolute value is closest to zero is set to zero, i.e., eliminated. This is repeated until a predefined percentage of attribute values is eliminated from the training set. The output of this elimination procedure is the pruned input training dataset. After the elimination phase is over, the standard SVM training is started with the pruned input dataset. This procedure, as a result, outputs the SVM model with sparse support vectors. 
Algorithm 1. Attribute Sparse Training SVM (AST-SVM) Algorithm. AST-SVM (DS, target_zv_pct)

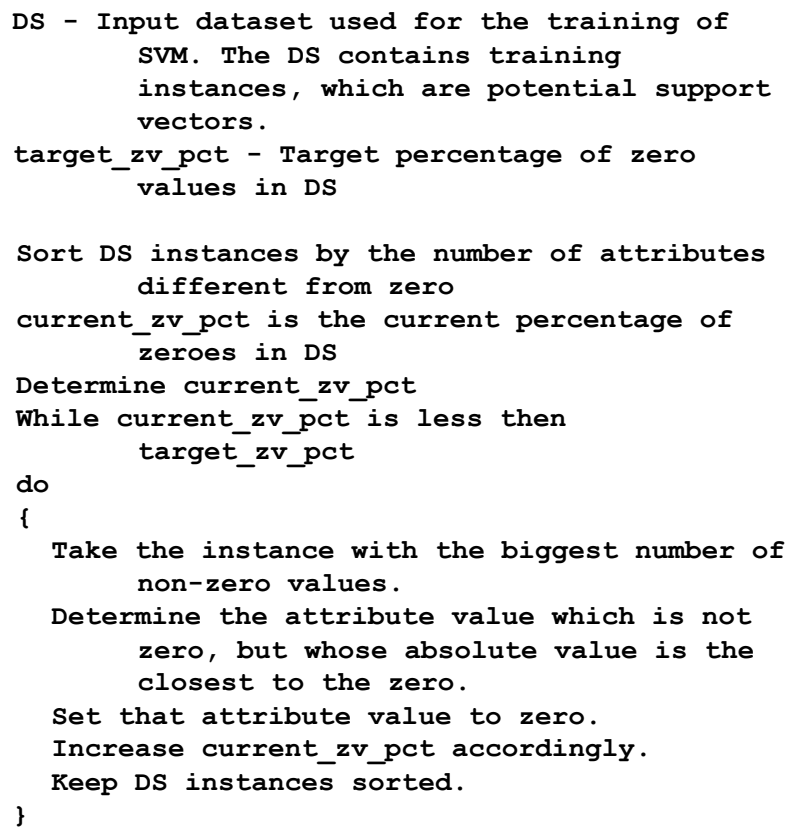

Run regular training SVM algorithm on modified DS and return SVM model as the result.

The listed algorithm uses an ordinary SVM training algorithm as one of its steps. We will call a model, which the ordinary algorithm outputs on the original, unmodified dataset, dense SVM model. The SVM model, which is the output of the AST-SVM algorithm, will be called "pruned SVM model". The dense model can contain some zero attributes in its support vectors. Please notice that the pruned SVM model always contains less non-zero attributes compared to the dense SVM model. However, that does not mean that the pruned SVM model always contains exactly target_zv_pct less non-zero attributes compared to the dense SVM model. The reason for this is that modification of the training dataset will change, which training instances will become support vectors. For example, the dense SVM model can have $0 \%$ of zeroes in its support vectors. The value of target_zv_pct can be set to $5 \%$. The modified training dataset will have $5 \%$ attribute values of the training instances set to zero. Nevertheless, after the ordinary SVM training algorithm is run on the modified training dataset, the resulting pruned SVM model can contain only $4 \%$ fewer attributes compared to the dense SVM model. The pruned SVM model can use more support vectors than the dense SVM model, but still has less non-zero attributes overall.

\section{HARDWARE ACCELERATOR FOR SPARSE SVMS}

The digital architecture, which can take advantage of the sparse SVM model, called "ASA-SVM" (Attribute Sparse Accelerator - SVM) is the modification of the "RMLC" (Reconfigurable Machine Learning Classifier) architecture, proposed in [20].

In the RMLC architecture, the SVM is implemented by splitting the sum calculation to several identical modules, each of which calculates only one part of the complete sum $V(x)$ as shown in Fig. 1.

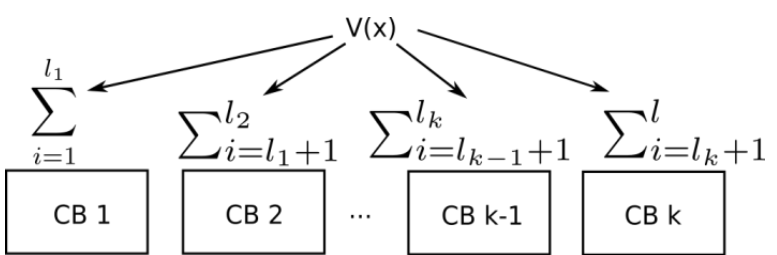

Fig. 1. Implementing SVM using RMLC architecture from [20].

In Fig. 1, there are K Computing Blocks (CB). Each CB calculates one part of the complete sum $V(x)$. CB 1 calculates sum up to $l_{1}^{\text {th }}$ support vector. The partial sum it calculates is then passed to the CB 2 module, alongside with the current input instance. The CB 2 module then calculates the next part of the complete sum from $\left(l_{1}+1\right)^{\text {th }}$ support vector to $l_{2}^{\text {th }}$ support vector and so on. The architecture is designed to achieve the high instance throughput by using pipelining. When the CB 1 module passes the partial sum of the first input instance to the CB 2 module, it immediately starts to calculate the partial sum using the next input instance. In the architecture with $\mathrm{K} \mathrm{CBs,} \mathrm{up} \mathrm{to} \mathrm{K}$ input instances can be processed at the same time.

The ASA-SVM architecture keeps only the SVM functionality part of the RMLC architecture and adds hardware support needed for the evaluation of sparse SVMs. The top-level block diagram of the ASA-SVM accelerator is shown in Fig. 2. The CBs are arranged in an array of identical pipeline stages. A configuration module (CM) reads the SVM configuration data from the main memory through the mm_rd interface and sends the data to a particular $\mathrm{CB}$. The configuration for each $\mathrm{CB}$ module consists of three parts. The first part is stored inside the support vector memory (SV_M) and it contains support vectors and Lagrange multipliers for the part of the complete sum, which that $\mathrm{CB}$ calculates. The second part contains increments stored in INC_M memory, which determine which attributes of the support vectors and the input instance should be processed. This enables skipping all multiplications that use zero attributes from the support vectors. The third part of the configuration is the samples of the specified non-linear function needed for the evaluation of the selected kernel function.

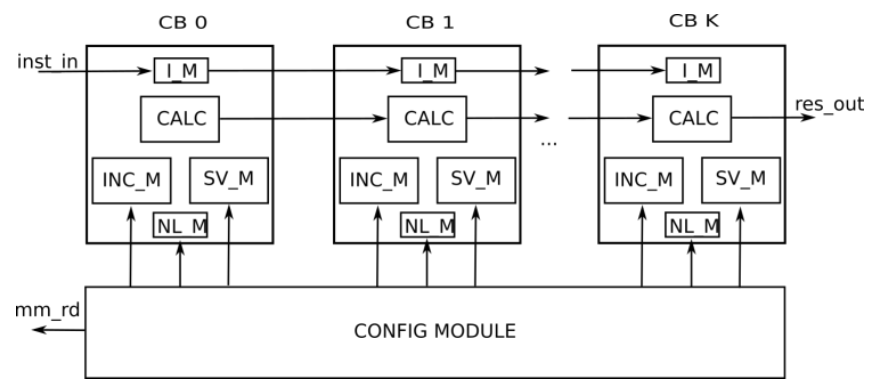

Fig. 2. Top-level block diagram of ASA-SVM accelerator.

In section II, the algorithm for the training of sparse SVMs was presented. The sparse SVMs have an advantage over ordinary SVM models since they contain fewer attributes with non-zero values. Let frac be the percentage of attributes, which are not zero. If the classification speed of the input instance is critical, then the sparse SVM models could be processed 1/frac times faster than the dense SVM models by skipping all product terms that use a zero-valued 
attribute. The hardware architecture needs to be designed to take advantage of this opportunity, and the ASA-SVM is the first SVM accelerator with this capability.

In Fig. 3, the skipping of product terms with zero-valued support vector attributes during kernel calculation is illustrated. Instead of storing the whole support vector in the memory, only non-zero values (NZV) are stored in the SV_M memory. Every NZV value corresponds to one increment value, which is stored in separate, INC_M memory. During the kernel calculation, for every NZV of the support vector, the corresponding value from the input instance is taken. All zero-valued attributes in the support vector are skipped during the kernel calculation evaluating the kernel faster. The second benefit of this technique is that usually less memory is needed for storing support vectors. Only the NZVs are stored with their corresponding increments. The increments can be coded with fewer bits. In the example, from Fig. 3, only 4 terms of the kernel calculation will be used instead of 12 . Also, only $4 \mathrm{NZV}$ will be stored, alongside with 4 increments.

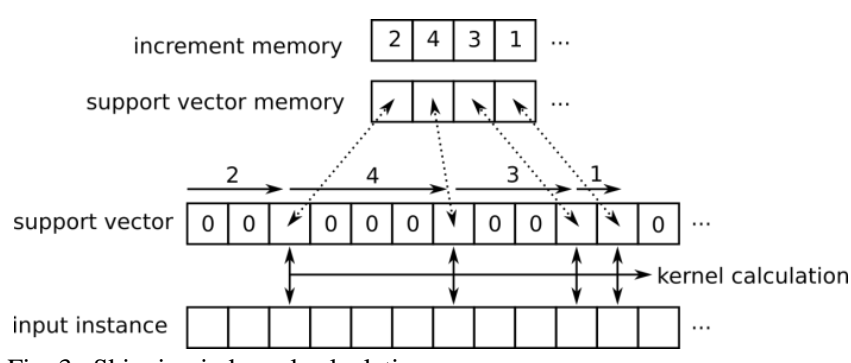

Fig. 3. Skipping in kernel calculation.

Each $\mathrm{CB}$ module is also pipelined. Figure 4 shows the architecture of the $\mathrm{CB}$. A control unit reads the values from the increment memory and calculates the effective address of the attributes. The effective address is used to read the current attribute from the input memory. In the SV memory, NZV values of support vectors are stored. The NZVs are read sequentially. The data read from the input memory and SV memory are sent for further processing to the appropriate functional unit.

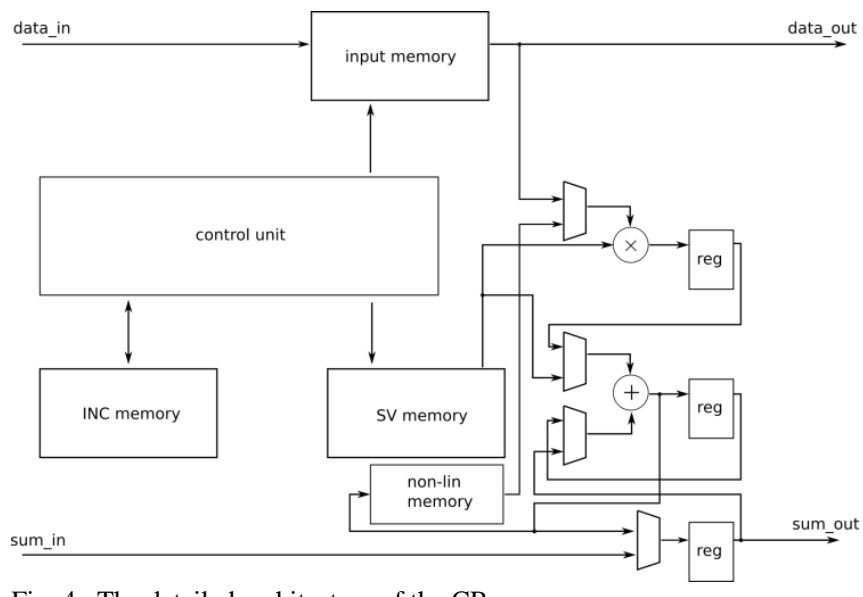

Fig. 4. The detailed architecture of the CB.

The control unit also controls the data selection multiplexers and enables signals of registers to configure pipelined data path required to implement the selected type of kernel needed for the classification. To perform the necessary computations, only one multiplier and adder are needed. After the vector calculation is finished, the final value is sent as an address to the non-lin memory. The value read from the non-lin memory is the kernel value for the current support vector and it is accumulated to the running sum after multiplication with the corresponding Lagrange multiplier stored in the SV memory. This process is then repeated for all support vectors stored in $\mathrm{CB}$.

The RMLC architecture from [22] has a processing time of "RB" (Reconfigurable Block) - $T_{R M L C}$

$$
T_{R M L C}=N_{s v}\left(N_{a t t r}+d\right) T_{c l k}
$$

where $N_{s v}$ is the number of SVs in a block, $N_{\text {attr }}$ is the number of attributes in the SV, the value $\mathrm{d}$ is 4 , depending on the type of kernel used in the SVM module, and $T_{c l k}$ is a period, on which the architecture works. The ASA-SVM architecture proposed in this paper has the processing time of one CB block - $T_{A S A-S V M}$

$$
T_{A S A-S V M}=\sum_{i=1}^{N_{S v}}\left(N_{n z v}^{i}+d\right) T_{c l k},
$$

where $N_{n z v}^{i}$ is the number of NZVs in the $i^{\text {th }} \mathrm{SV}$ of the CB. The value of $N_{n z v}^{i}$ is always smaller than $N_{a t t r}$, so the processing speed of ASA-SVM is always greater than RMLC's.

The AST-SVM algorithm is designed to keep the number of NZVs evenly distributed along all support vectors. For every dataset, there is a threshold, when the maximum difference between $N_{n z v}^{i}$ values will be 1 . Let $N_{n z v}$ be $\max \left(N_{n z v}^{i}\right)$. Processing time of ASA-SVM architecture always satisfies the following condition

$$
T_{A S A-S V M} \leq N_{s v}\left(N_{n z v}+d\right) T_{c l k} .
$$

The speed-up $S$ of the ASA-SVM architecture, compared to RMLC, can be calculated as

$$
S=\frac{T_{R M L C}}{T_{A S A-S V M}} \geq \frac{N_{a t t r}+d}{N_{n z v}+d} .
$$

For large datasets, where $N_{\text {attr }} \gg>d$ and $N_{n z v}>>d$, the speed-up equation can be approximated as

$$
S \approx \frac{N_{a t r}}{N_{n z v}}=\frac{1}{f r a c} .
$$

From the approximate equation for speed-up, it is clear that the efficiency of the ASA-SVM is directly proportional to the amount of pruning achieved during the training of the sparse SVM model.

\section{EXPERIMENTAL RESULTS}

Xilinx Vivado Design Suite is used for the development of the ASA-SVM architecture. The default values for synthesis and implementation settings were set. Zynq Ultrascale+ MPSoC ZCU102 Evaluation Board [21] was a 
test platform, on which experiments were done.

The training of SVM models after pruning of the datasets was done using the LIBSVM library [22]. All standard procedures from the library were used with default parameters.

The ability of the AST-SVM algorithm to compress SVM classifiers was tested on several datasets from the LIBSVM dataset page [23]. The datasets of various sizes and characteristics were selected.

For every dataset, 19 pruning factors were used as inputs for the AST-SVM algorithm. The targeted pruning factors ranged from $5 \%$ to $95 \%$ with steps of $5 \%$. For every pruning factor, the dataset was pruned, and then the LIBSVM training procedure was called to create sparse SVM. The accuracy of the resulting sparse SVM model and its size was then determined. In all cases, if the dataset was split into training and validation sets, the training set was used for training, and the accuracy was measured on the validation dataset. In case when the dataset has not been split, the whole dataset was used for training, and also for the accuracy measurement. Table I presents the major characteristics of the datasets that were used in the experiments.

TABLE I. DATASETS' CHARACTERISTICS.

\begin{tabular}{|c|c|c|c|}
\hline Dataset name & Short name & Attributes & Instances \\
\hline Wisconsin Breast Cancer & bcancer & 10 & 683 \\
\hline Pima Indians Diabetes & diabetes & 8 & 768 \\
\hline Glass Identification & glass & 9 & 214 \\
\hline Heart Disease & heart & 13 & 270 \\
\hline Wine Recognition & wine & 13 & 178 \\
\hline Mushrooms & mush & 112 & 8124 \\
\hline USPS & usps & 256 & 7291 \\
\hline Poker Hand & poker & 10 & 25010 \\
\hline MNIST & mnist & 780 & 60000 \\
\hline CIFAR10 & cifar & 3072 & 50000 \\
\hline SVHN & svhn & 3072 & 73257 \\
\hline
\end{tabular}

In the following Table II-Table XII and Fig. 5-Fig. 15, the results of the experiments are shown. The tables show how much it is possible to prune the SVM model trained using the AST-SVM algorithm on the given dataset. Also, real size reductions are shown.

TABLE II. BENCHMARKING RESULTS - BCANCER

\begin{tabular}{|c|c|c|c|c|c|c|}
\hline Target & ACC & NZV & ZV & Reduction & Speed-up & Memory \\
\hline 0 & 97.2182 & 814 & 0 & 1.00 & 1.00 & 1.25 \\
\hline 0.05 & 97.0717 & 821 & 26 & 1.01 & 0.99 & 1.26 \\
\hline 0.1 & 97.2182 & 750 & 75 & 0.92 & 1.06 & 1.15 \\
\hline 0.15 & 97.0717 & 741 & 106 & 0.91 & 1.07 & 1.14 \\
\hline 0.2 & 96.6325 & 710 & 159 & 0.87 & 1.10 & 1.09 \\
\hline 0.25 & 96.3397 & 720 & 204 & 0.88 & 1.09 & 1.11 \\
\hline 0.3 & 96.4861 & 648 & 243 & 0.80 & 1.17 & 1.00 \\
\hline 0.35 & 96.3397 & 668 & 300 & 0.82 & 1.15 & 1.03 \\
\hline 0.4 & 96.0469 & 623 & 356 & 0.77 & 1.20 & 0.96 \\
\hline 0.45 & 96.1933 & 628 & 417 & 0.77 & 1.20 & 0.96 \\
\hline 0.5 & 95.754 & 570 & 475 & 0.70 & 1.27 & 0.88 \\
\hline 0.55 & 95.6076 & 561 & 539 & 0.69 & 1.29 & 0.86 \\
\hline 0.6 & 95.4612 & 495 & 594 & 0.61 & 1.39 & 0.76 \\
\hline 0.65 & 95.022 & 518 & 736 & 0.64 & 1.35 & 0.80 \\
\hline 0.7 & 94.7291 & 495 & 869 & 0.61 & 1.39 & 0.76 \\
\hline 0.75 & 94.2899 & 547 & 1213 & 0.67 & 1.31 & 0.84 \\
\hline 0.8 & 94.7291 & 527 & 1409 & 0.65 & 1.34 & 0.81 \\
\hline 0.85 & 91.9473 & 756 & 2797 & 0.93 & 1.05 & 1.16 \\
\hline 0.9 & 76.2811 & 665 & 2998 & 0.82 & 1.15 & 1.02 \\
\hline 0.95 & 89.1654 & 718 & 4551 & 0.88 & 1.09 & 1.10 \\
\hline
\end{tabular}

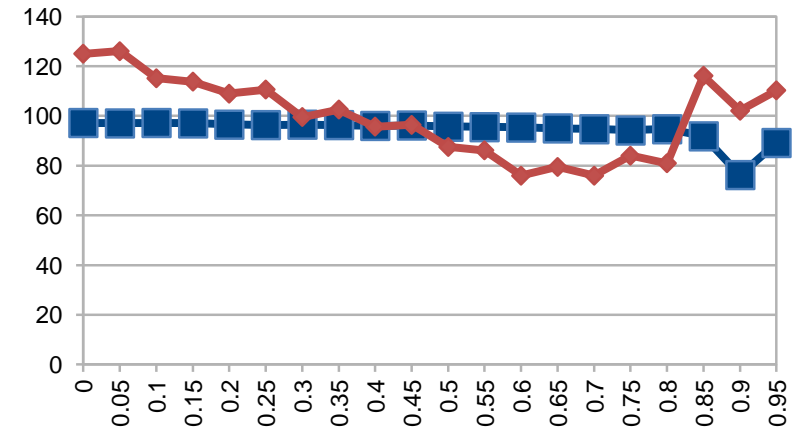

Fig. 5. Pruning-accuracy graph for bcancer.

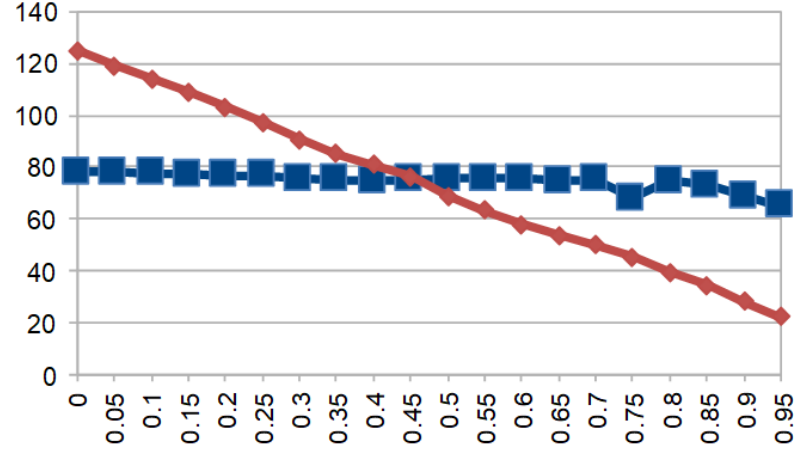

Fig. 6. Pruning-accuracy graph for diabetes. 
TABLE III. BENCHMARKING RESULTS - DIABETES.

\begin{tabular}{|c|c|c|c|c|c|c|}
\hline Target & ACC & $\mathrm{NZV}$ & $\mathrm{ZV}$ & Reduction & Speed-up & Memory \\
\hline 0 & 77.9948 & 4232 & 7 & 1.00 & 1.00 & 1.25 \\
\hline 0.05 & 78.125 & 4040 & 190 & 0.95 & 1.03 & 1.19 \\
\hline 0.1 & 77.474 & 3872 & 376 & 0.91 & 1.06 & 1.14 \\
\hline 0.15 & 77.2135 & 3703 & 563 & 0.88 & 1.09 & 1.09 \\
\hline 0.2 & 76.6927 & 3502 & 746 & 0.83 & 1.13 & 1.03 \\
\hline 0.25 & 76.8229 & 3299 & 949 & 0.78 & 1.17 & 0.97 \\
\hline 0.3 & 75.5208 & 3074 & 1111 & 0.73 & 1.22 & 0.91 \\
\hline 0.35 & 75.2604 & 2892 & 1302 & 0.68 & 1.27 & 0.85 \\
\hline 0.4 & 74.8698 & 2752 & 1541 & 0.65 & 1.31 & 0.81 \\
\hline 0.45 & 75.2604 & 2584 & 1736 & 0.61 & 1.35 & 0.76 \\
\hline 0.5 & 75.3906 & 2340 & 1881 & 0.55 & 1.43 & 0.69 \\
\hline 0.55 & 75.5208 & 2144 & 2131 & 0.51 & 1.49 & 0.63 \\
\hline 0.6 & 75.3906 & 1965 & 2274 & 0.46 & 1.56 & 0.58 \\
\hline 0.65 & 74.6094 & 1826 & 2602 & 0.43 & 1.61 & 0.54 \\
\hline 0.7 & 75 & 1700 & 2935 & 0.40 & 1.66 & 0.50 \\
\hline 0.75 & 68.099 & 1540 & 3086 & 0.36 & 1.74 & 0.45 \\
\hline 0.8 & 74.7396 & 1341 & 3483 & 0.32 & 1.84 & 0.40 \\
\hline 0.85 & 73.1771 & 1167 & 3702 & 0.28 & 1.93 & 0.34 \\
\hline 0.9 & 68.6198 & 949 & 3893 & 0.22 & 2.07 & 0.28 \\
\hline 0.95 & 65.1042 & 751 & 4091 & 0.18 & 2.22 & 0.22 \\
\hline
\end{tabular}

TABLE IV. BENCHMARKING RESULTS - GLASS.

\begin{tabular}{|c|c|c|c|c|c|c|}
\hline Target & ACC & NZV & ZV & Reduction & Speed-up & Memory \\
\hline 0 & 59.3458 & 2334 & 536 & 1.00 & 1.15 & 1.02 \\
\hline 0.05 & 56.0748 & 2234 & 608 & 0.96 & 1.18 & 0.97 \\
\hline 0.1 & 58.8785 & 2139 & 703 & 0.92 & 1.21 & 0.93 \\
\hline 0.15 & 60.2804 & 2059 & 797 & 0.88 & 1.24 & 0.90 \\
\hline 0.2 & 56.5421 & 1973 & 883 & 0.85 & 1.28 & 0.86 \\
\hline 0.25 & 61.6822 & 1885 & 985 & 0.81 & 1.31 & 0.82 \\
\hline 0.3 & 52.8037 & 1799 & 1071 & 0.77 & 1.35 & 0.78 \\
\hline 0.35 & 58.8785 & 1692 & 1164 & 0.72 & 1.40 & 0.74 \\
\hline 0.4 & 60.2804 & 1629 & 1255 & 0.70 & 1.43 & 0.71 \\
\hline 0.45 & 63.0841 & 1528 & 1342 & 0.65 & 1.48 & 0.67 \\
\hline 0.5 & 53.7383 & 1432 & 1424 & 0.61 & 1.53 & 0.62 \\
\hline 0.55 & 54.2056 & 1362 & 1550 & 0.58 & 1.57 & 0.59 \\
\hline 0.6 & 50.9346 & 1259 & 1639 & 0.54 & 1.64 & 0.55 \\
\hline 0.65 & 47.1963 & 1178 & 1720 & 0.50 & 1.69 & 0.51 \\
\hline 0.7 & 43.9252 & 1072 & 1840 & 0.46 & 1.77 & 0.47 \\
\hline 0.75 & 45.7944 & 1017 & 1909 & 0.44 & & 1.81 \\
\hline 0.8 & 45.3271 & 940 & 2056 & 0.40 & 1.87 & 0.44 \\
\hline 0.85 & 35.514 & 852 & 2116 & 0.37 & & 0.41 \\
\hline 0.9 & 35.9813 & 751 & 2175 & 0.32 & & 0.37 \\
\hline 0.95 & 33.6449 & 645 & 2211 & 0.28 & 2.05 & 0.33 \\
\hline
\end{tabular}

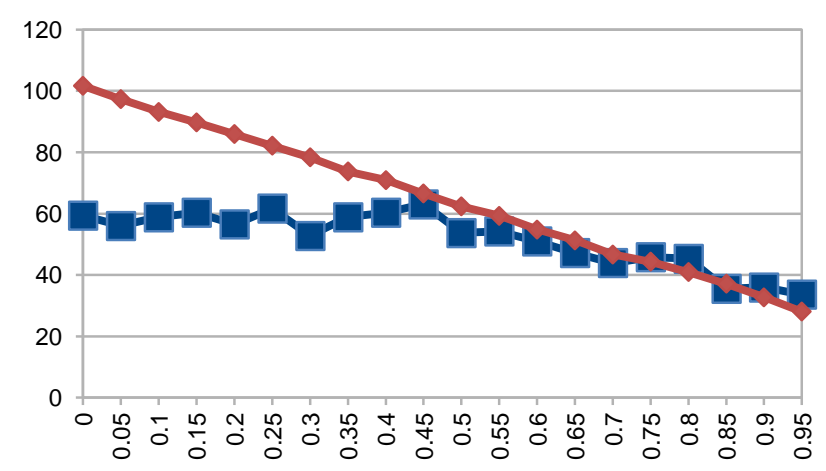

Fig. 7. Pruning-accuracy graph for glass.

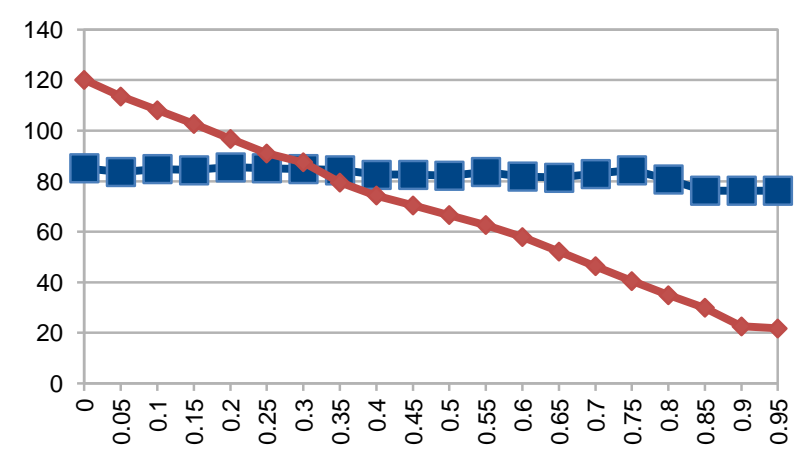

Fig. 8. Pruning-accuracy graph for heart. 
TABLE V. BENCHMARKING RESULTS - HEART.

\begin{tabular}{|c|c|c|c|c|c|c|}
\hline Target & ACC & NZV & ZV & Reduction & Speed-up & Memory \\
\hline 0 & 85.1852 & 1668 & 68 & 1.00 & 1.03 & 1.20 \\
\hline 0.05 & 83.7037 & 1576 & 132 & 0.94 & 1.08 & 1.13 \\
\hline 0.1 & 84.8148 & 1501 & 207 & 0.90 & 1.12 & 1.08 \\
\hline 0.15 & 84.4444 & 1426 & 282 & 0.85 & 1.16 & 1.03 \\
\hline 0.2 & 85.5556 & 1343 & 365 & 0.81 & 1.21 & 0.97 \\
\hline 0.25 & 85.1852 & 1263 & 431 & 0.76 & 1.26 & 0.91 \\
\hline 0.3 & 84.8148 & 1214 & 522 & 0.73 & 1.30 & 0.87 \\
\hline 0.35 & 84.4444 & 1104 & 590 & 0.66 & 1.39 & 0.79 \\
\hline 0.4 & 82.5926 & 1032 & 662 & 0.62 & 1.45 & 0.74 \\
\hline 0.45 & 82.5926 & 978 & 758 & 0.59 & 1.50 & 0.70 \\
\hline 0.5 & 82.2222 & 924 & 854 & 0.55 & 1.56 & 0.67 \\
\hline 0.55 & 83.7037 & 870 & 964 & 0.52 & 1.62 & 0.63 \\
\hline 0.6 & 81.8519 & 804 & 1072 & 0.48 & 1.70 & 0.58 \\
\hline 0.65 & 81.4815 & 725 & 1151 & 0.43 & 1.80 & 0.52 \\
\hline 0.7 & 82.963 & 643 & 1247 & 0.39 & 1.93 & 0.46 \\
\hline 0.75 & 84.4444 & 562 & 1342 & 0.34 & 2.07 & 0.40 \\
\hline 0.8 & 80.7407 & 485 & 1489 & 0.29 & 2.23 & 0.35 \\
\hline 0.85 & 76.2963 & 415 & 1643 & 0.25 & 2.39 & 0.30 \\
\hline 0.9 & 76.2963 & 314 & 1702 & 0.19 & 2.68 & 0.23 \\
\hline 0.95 & 76.2963 & 302 & 2526 & 0.18 & 2.71 & 0.22 \\
\hline & & & & & & \\
\hline
\end{tabular}

TABLE VI. BENCHMARKING RESULTS - WINE.

\begin{tabular}{|c|c|c|c|c|c|c|}
\hline Target & ACC & NZV & ZV & Reduction & Speed-up & Memory \\
\hline 0 & 98.3146 & 1351 & 74 & 1.00 & 1.04 & 1.19 \\
\hline 0.05 & 98.3146 & 1287 & 138 & 0.95 & 1.08 & 1.13 \\
\hline 0.1 & 98.8764 & 1241 & 199 & 0.92 & 1.11 & 1.09 \\
\hline 0.15 & 98.3146 & 1183 & 257 & 0.88 & 1.15 & 1.04 \\
\hline 0.2 & 99.4382 & 1138 & 332 & 0.84 & 1.18 & 1.00 \\
\hline 0.25 & 98.8764 & 1056 & 384 & 0.78 & 1.25 & 0.93 \\
\hline 0.3 & 98.8764 & 1003 & 452 & 0.74 & 1.29 & 0.88 \\
\hline 0.35 & 98.8764 & 939 & 501 & 0.70 & 1.35 & 0.82 \\
\hline 0.4 & 97.7528 & 876 & 564 & 0.65 & 1.42 & 0.77 \\
\hline 0.45 & 97.7528 & 824 & 646 & 0.61 & 1.48 & 0.72 \\
\hline 0.5 & 97.7528 & 799 & 731 & 0.59 & 1.51 & 0.70 \\
\hline 0.55 & 96.6292 & 750 & 825 & 0.56 & 1.57 & 0.66 \\
\hline 0.6 & 96.0674 & 695 & 895 & 0.51 & 1.64 & 0.61 \\
\hline 0.65 & 96.0674 & 629 & 946 & 0.47 & 1.75 & 0.55 \\
\hline 0.7 & 94.9438 & 601 & 1079 & 0.44 & 1.79 & 0.53 \\
\hline 0.75 & 96.0674 & 545 & 1165 & 0.40 & 1.89 & 0.48 \\
\hline 0.8 & 93.2584 & 548 & 1462 & 0.41 & 1.89 & 0.48 \\
\hline 0.85 & 92.1348 & 517 & 1673 & 0.38 & 1.95 & 0.45 \\
\hline 0.9 & 94.382 & 502 & 1958 & 0.37 & 1.98 & 0.44 \\
\hline 0.95 & 70.2247 & 424 & 2141 & 0.31 & 2.16 & 0.37 \\
\hline
\end{tabular}

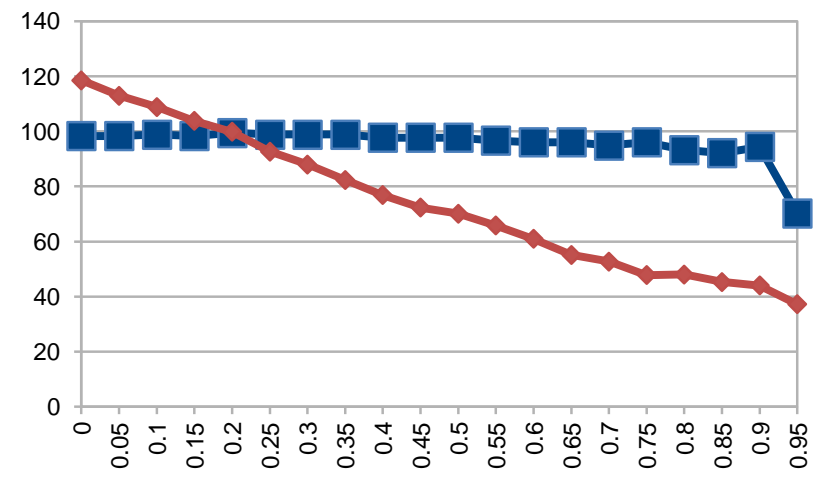

Fig. 9. Pruning-accuracy graph for wine.

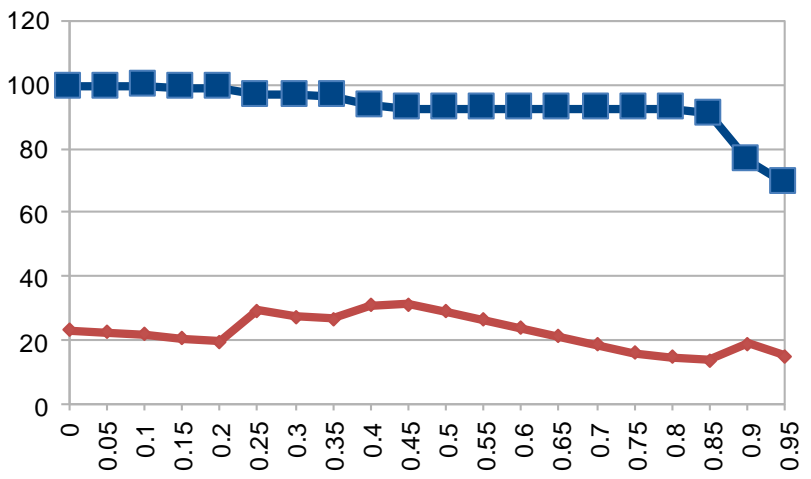

Fig. 10. Pruning-accuracy/size graph for mush. 
TABLE VII. BENCHMARKING RESULTS - MUSH.

\begin{tabular}{|c|c|c|c|c|c|c|}
\hline Target & ACC & $\mathrm{NZV}$ & $\mathrm{ZV}$ & Reduction & Speed-up & Memory \\
\hline 0 & 99.2122 & 17766 & 77832 & 1.00 & 4.67 & 0.23 \\
\hline 0.05 & 99.2614 & 17120 & 79608 & 0.96 & 4.82 & 0.22 \\
\hline 0.1 & 99.5076 & 16701 & 82626 & 0.94 & 4.92 & 0.22 \\
\hline 0.15 & 99.0153 & 15732 & 83030 & 0.89 & 5.17 & 0.21 \\
\hline 0.2 & 99.0153 & 14908 & 84193 & 0.84 & 5.40 & 0.19 \\
\hline 0.25 & 96.8488 & 22336 & 135412 & 1.26 & 3.85 & 0.29 \\
\hline 0.3 & 96.8488 & 21000 & 137200 & 1.18 & 4.06 & 0.27 \\
\hline 0.35 & 96.4549 & 20551 & 145333 & 1.16 & 4.13 & 0.27 \\
\hline 0.4 & 93.4023 & 23660 & 182000 & 1.33 & 3.66 & 0.31 \\
\hline 0.45 & 92.6145 & 24023 & 202203 & 1.35 & 3.61 & 0.31 \\
\hline 0.5 & 92.4175 & 22055 & 204510 & 1.24 & 3.89 & 0.29 \\
\hline 0.55 & 92.4175 & 20129 & 207340 & 1.13 & 4.21 & 0.26 \\
\hline 0.6 & 92.4175 & 18098 & 209145 & 1.02 & 4.60 & 0.24 \\
\hline 0.65 & 92.4175 & 16184 & 212415 & 0.91 & 5.05 & 0.21 \\
\hline 0.7 & 92.4175 & 14273 & 216134 & 0.80 & 5.60 & 0.19 \\
\hline 0.75 & 92.4175 & 12329 & 219886 & 0.69 & 6.29 & 0.16 \\
\hline 0.8 & 92.4668 & 11279 & 243649 & 0.63 & 6.74 & 0.15 \\
\hline 0.85 & 90.9404 & 10431 & 284273 & 0.59 & 7.15 & 0.14 \\
\hline 0.9 & 76.2186 & 14553 & 533610 & 0.82 & 5.51 & 0.19 \\
\hline 0.95 & 69.03 & 11607 & 644245 & 0.65 & 6.59 & 0.15 \\
\hline
\end{tabular}

TABLE VIII. BENCHMARKING RESULTS - USPS.

\begin{tabular}{|c|c|c|c|c|c|c|}
\hline Target & ACC & NZV & ZV & Reduction & Speed-up & Memory \\
\hline 0 & 91.7289 & 668262 & 15438 & 1.00 & 1.02 & 1.22 \\
\hline 0.05 & 91.5795 & 637712 & 48638 & 0.95 & 1.07 & 1.17 \\
\hline 0.1 & 91.7788 & 607076 & 82189 & 0.91 & 1.12 & 1.11 \\
\hline 0.15 & 91.7289 & 593198 & 119652 & 0.89 & 1.15 & 1.08 \\
\hline 0.2 & 91.8784 & 563577 & 155368 & 0.84 & 1.21 & 1.03 \\
\hline 0.25 & 91.6791 & 535909 & 192576 & 0.80 & 1.27 & 0.98 \\
\hline 0.3 & 91.1809 & 525131 & 239129 & 0.79 & 1.30 & 0.96 \\
\hline 0.35 & 89.3373 & 488840 & 276215 & 0.73 & 1.39 & 0.89 \\
\hline 0.4 & 85.999 & 464114 & 321611 & 0.69 & 1.46 & 0.85 \\
\hline 0.45 & 80.5182 & 446167 & 376658 & 0.67 & 1.52 & 0.82 \\
\hline 0.5 & 76.8809 & 402136 & 411149 & 0.60 & 1.68 & 0.74 \\
\hline 0.55 & 73.8416 & 396460 & 492880 & 0.59 & 1.71 & 0.72 \\
\hline 0.6 & 71.4001 & 366015 & 554860 & 0.55 & 1.84 & 0.67 \\
\hline 0.65 & 67.713 & 331885 & 617345 & 0.50 & 2.03 & 0.61 \\
\hline 0.7 & 64.3747 & 302437 & 701118 & 0.45 & 2.22 & 0.55 \\
\hline 0.75 & 60.289 & 268751 & 794429 & 0.40 & 2.48 & 0.49 \\
\hline 0.8 & 56.2033 & 236489 & 920236 & 0.35 & 2.81 & 0.43 \\
\hline 0.85 & 53.0144 & 203762 & 1097918 & 0.30 & 3.24 & 0.37 \\
\hline 0.9 & 43.4479 & 161777 & 1319573 & 0.24 & 4.03 & 0.30 \\
\hline 0.95 & 34.3797 & 102880 & 1517065 & 0.15 & 6.11 & 0.19 \\
\hline
\end{tabular}

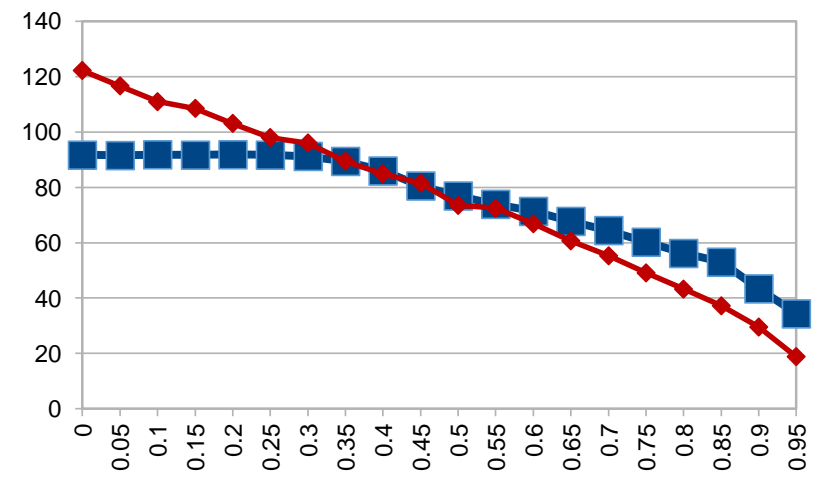

Fig. 11. Pruning-accuracy/size graph for USPS.

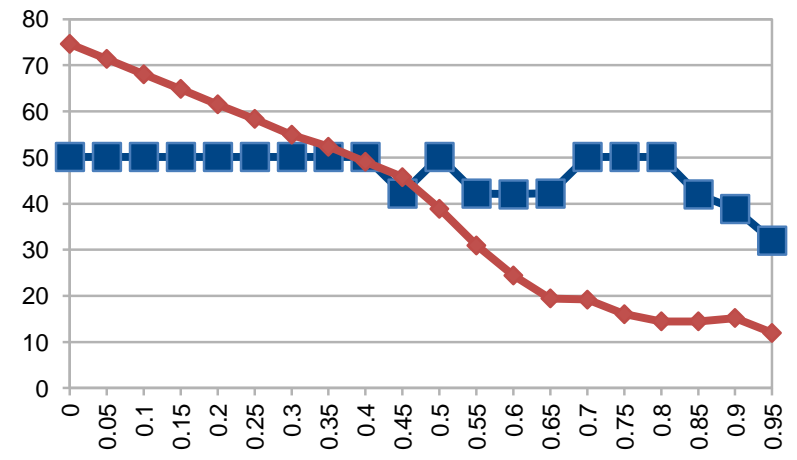

Fig. 12. Pruning-accuracy/size graph for poker. 
TABLE IX. BENCHMARKING RESULTS - POKER.

\begin{tabular}{|c|c|c|c|c|c|c|}
\hline Target & ACC & NZV & ZV & Reduction & Speed-up & Memory \\
\hline 0 & 50.1209 & 263110 & 177766 & 1.00 & 1.40 & 0.75 \\
\hline 0.05 & 50.1209 & 251772 & 189237 & 0.96 & 1.44 & 0.71 \\
\hline 0.1 & 50.1209 & 240094 & 201181 & 0.91 & 1.48 & 0.68 \\
\hline 0.15 & 50.1209 & 228739 & 212593 & 0.87 & 1.52 & 0.65 \\
\hline 0.2 & 50.1209 & 216969 & 224534 & 0.82 & 1.57 & 0.62 \\
\hline 0.25 & 50.1209 & 205778 & 235972 & 0.78 & 1.62 & 0.58 \\
\hline 0.3 & 50.1209 & 193716 & 247730 & 0.74 & 1.67 & 0.55 \\
\hline 0.35 & 50.1209 & 184629 & 263258 & 0.70 & 1.71 & 0.52 \\
\hline 0.4 & 50.1209 & 173009 & 276816 & 0.66 & 1.77 & 0.49 \\
\hline 0.45 & 42.2479 & 161326 & 291083 & 0.61 & 1.83 & 0.46 \\
\hline 0.5 & 50.1209 & 137262 & 276273 & 0.52 & 1.97 & 0.39 \\
\hline 0.55 & 42.1724 & 109096 & 243696 & 0.41 & 2.16 & 0.31 \\
\hline 0.6 & 42.0758 & 86266 & 220869 & 0.33 & 2.35 & 0.24 \\
\hline 0.65 & 42.2498 & 68480 & 190110 & 0.26 & 2.52 & 0.19 \\
\hline 0.7 & 50.1209 & 67689 & 224588 & 0.26 & 2.53 & 0.19 \\
\hline 0.75 & 50.1209 & 56531 & 211977 & 0.21 & 2.65 & 0.16 \\
\hline 0.8 & 50.1209 & 51010 & 224357 & 0.19 & 2.71 & 0.14 \\
\hline 0.85 & 42.0069 & 51019 & 299227 & 0.19 & 2.71 & 0.14 \\
\hline 0.9 & 38.8308 & 53604 & 381591 & 0.20 & 2.68 & 0.15 \\
\hline 0.95 & 32.0346 & 42312 & 393415 & 0.16 & 2.82 & 0.12 \\
\hline
\end{tabular}

TABLE X. BENCHMARKING RESULTS - MNIST.

\begin{tabular}{|c|c|c|c|c|c|c|}
\hline Target & ACC & NZV & ZV & Reduction & Speed-up & Memory \\
\hline 0 & 93.13 & 3440972 & 14928526 & 1.00 & 5.22 & 0.23 \\
\hline 0.05 & 93.19 & 3327687 & 15075738 & 0.97 & 5.40 & 0.23 \\
\hline 0.1 & 93.17 & 3208746 & 15290148 & 0.93 & 5.59 & 0.22 \\
\hline 0.15 & 93.14 & 3087787 & 15586265 & 0.90 & 5.80 & 0.21 \\
\hline 0.2 & 93.1 & 2971934 & 15994048 & 0.86 & 6.02 & 0.20 \\
\hline 0.25 & 93.04 & 2849282 & 16457548 & 0.83 & 6.27 & 0.19 \\
\hline 0.3 & 92.94 & 2731426 & 17046437 & 0.79 & 6.53 & 0.19 \\
\hline 0.35 & 92.66 & 2621558 & 17803285 & 0.76 & 6.80 & 0.18 \\
\hline 0.4 & 92.23 & 2521740 & 18763902 & 0.73 & 7.06 & 0.17 \\
\hline 0.45 & 91.91 & 2422939 & 19861701 & 0.70 & 7.34 & 0.16 \\
\hline 0.5 & 91.65 & 2328891 & 21211033 & 0.68 & 7.62 & 0.16 \\
\hline 0.55 & 91.31 & 2238182 & 22831250 & 0.65 & 7.92 & 0.15 \\
\hline 0.6 & 90.81 & 2131474 & 24569118 & 0.62 & 8.30 & 0.15 \\
\hline 0.65 & 90.28 & 2028023 & 26782833 & 0.59 & 8.70 & 0.14 \\
\hline 0.7 & 89.47 & 1909403 & 29330857 & 0.55 & 9.22 & 0.13 \\
\hline 0.75 & 87.18 & 1765278 & 32266078 & 0.51 & 9.93 & 0.12 \\
\hline 0.8 & 83.78 & 1593953 & 35528837 & 0.46 & 10.94 & 0.11 \\
\hline 0.85 & 76.85 & 1370830 & 38833718 & 0.40 & 12.60 & 0.09 \\
\hline 0.9 & 71.66 & 1118770 & 42109334 & 0.33 & 15.22 & 0.08 \\
\hline 0.95 & 52.75 & 851145 & 45191695 & 0.25 & 19.53 & 0.06 \\
\hline
\end{tabular}

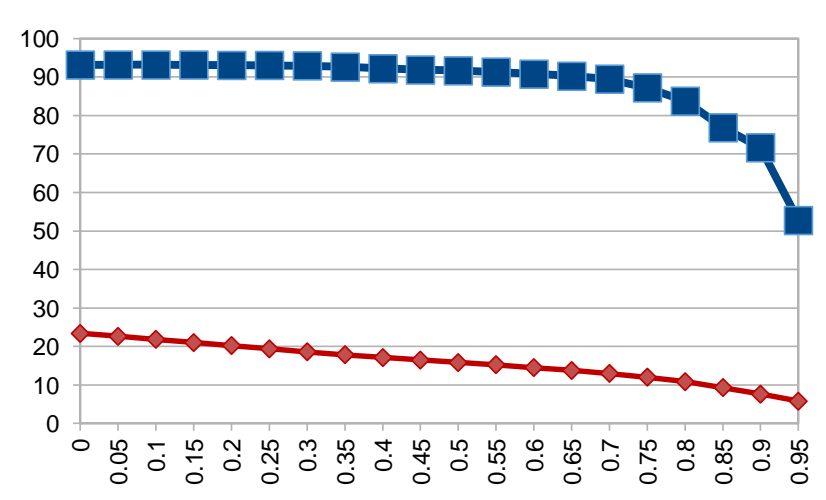

Fig. 13. Pruning-accuracy/size graph for MNIST.

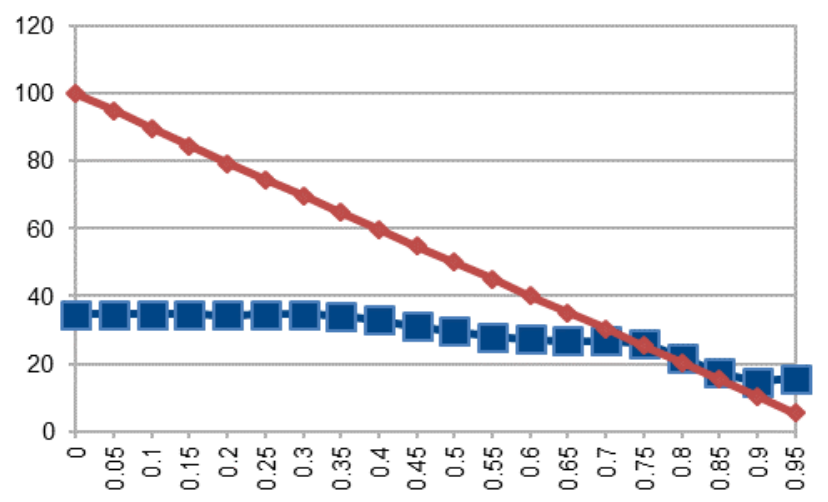

Fig. 14. Pruning-accuracy/size graph for CIFAR. 
TABLE XI. BENCHMARKING RESULTS - CIFAR.

\begin{tabular}{|c|c|c|c|c|c|c|}
\hline Target & ACC & NZV & ZV & Reduction & Speed-up & Memory \\
\hline 0 & 34.54 & 146841985 & 519164 & 1.00 & 1.00 & 1.25 \\
\hline 0.05 & 34.52 & 139261741 & 7840604 & 0.95 & 1.06 & 1.18 \\
\hline 0.1 & 34.66 & 131545686 & 15112995 & 0.90 & 1.12 & 1.12 \\
\hline 0.15 & 34.69 & 124122919 & 22384793 & 0.85 & 1.19 & 1.05 \\
\hline 0.2 & 34.36 & 116487853 & 29582357 & 0.79 & 1.26 & 0.99 \\
\hline 0.25 & 34.57 & 109412240 & 36910612 & 0.75 & 1.35 & 0.93 \\
\hline 0.3 & 34.71 & 102270755 & 44246200 & 0.70 & 1.44 & 0.87 \\
\hline 0.35 & 34.09 & 95169529 & 51633959 & 0.65 & 1.55 & 0.81 \\
\hline 0.4 & 32.94 & 87929910 & 58975251 & 0.60 & 1.67 & 0.75 \\
\hline 0.45 & 31.13 & 80650407 & 66300969 & 0.55 & 1.83 & 0.68 \\
\hline 0.5 & 29.61 & 73396121 & 73660009 & 0.50 & 2.01 & 0.62 \\
\hline 0.55 & 28.03 & 66172232 & 81078001 & 0.45 & 2.22 & 0.56 \\
\hline 0.6 & 27.22 & 58899163 & 88468148 & 0.40 & 2.50 & 0.50 \\
\hline 0.65 & 26.75 & 51637803 & 95911287 & 0.35 & 2.85 & 0.44 \\
\hline 0.7 & 26.79 & 44419751 & 103514464 & 0.30 & 3.31 & 0.38 \\
\hline 0.75 & 26.03 & 37189070 & 111228862 & 0.25 & 3.95 & 0.32 \\
\hline 0.8 & 21.81 & 29947728 & 119132619 & 0.20 & 4.90 & 0.25 \\
\hline 0.85 & 17.2 & 22699792 & 127418852 & 0.15 & 6.45 & 0.19 \\
\hline 0.9 & 14.61 & 15343490 & 135699454 & 0.10 & 9.50 & 0.13 \\
\hline 0.95 & 15.64 & 7944104 & 144679393 & 0.05 & 18.14 & 0.07 \\
\hline
\end{tabular}

TABLE XII. BENCHMARKING RESULTS - SVHN.

\begin{tabular}{|c|c|c|c|c|c|c|}
\hline Target & ACC & $\mathbf{N Z V}$ & $\mathbf{Z V}$ & Reduction & Speed-up & Memory \\
\hline 0 & 13.7754 & 205086232 & 191555 & 1.00 & 1.00 & 1.25 \\
\hline 0.05 & 13.9098 & 194957987 & 10421473 & 0.95 & 1.05 & 1.19 \\
\hline 0.1 & 14.2555 & 185743497 & 20763609 & 0.91 & 1.11 & 1.13 \\
\hline 0.15 & 14.6512 & 176805075 & 31288746 & 0.86 & 1.16 & 1.08 \\
\hline 0.2 & 15.7345 & 168223458 & 42101007 & 0.82 & 1.22 & 1.02 \\
\hline 0.25 & 16.9215 & 160214044 & 53407091 & 0.78 & 1.28 & 0.98 \\
\hline 0.3 & 18.5925 & 151307562 & 64803021 & 0.74 & 1.36 & 0.92 \\
\hline 0.35 & 20.3058 & 141372009 & 76026432 & 0.69 & 1.45 & 0.86 \\
\hline 0.4 & 20.8666 & 131534404 & 87530858 & 0.64 & 1.56 & 0.80 \\
\hline 0.45 & 20.8052 & 121352380 & 99056198 & 0.59 & 1.69 & 0.74 \\
\hline 0.5 & 20.2597 & 110768233 & 110447567 & 0.54 & 1.85 & 0.67 \\
\hline 0.55 & 20.1099 & 99798629 & 121546573 & 0.49 & 2.05 & 0.61 \\
\hline 0.6 & 20.179 & 88701366 & 132486705 & 0.43 & 2.31 & 0.54 \\
\hline 0.65 & 20.3365 & 77667657 & 143501928 & 0.38 & 2.64 & 0.47 \\
\hline 0.7 & 20.3288 & 66544984 & 154301096 & 0.32 & 3.08 & 0.41 \\
\hline 0.75 & 20.3941 & 55487560 & 165164417 & 0.27 & 3.69 & 0.34 \\
\hline 0.8 & 20.4057 & 44471245 & 176088302 & 0.22 & 4.59 & 0.27 \\
\hline 0.85 & 20.2674 & 33512888 & 187262329 & 0.16 & 6.08 & 0.20 \\
\hline 0.9 & 19.3838 & 22619933 & 199199743 & 0.11 & 8.98 & 0.14 \\
\hline 0.95 & 18.5426 & 11648912 & 211649644 & 0.06 & 17.25 & 0.07 \\
\hline
\end{tabular}

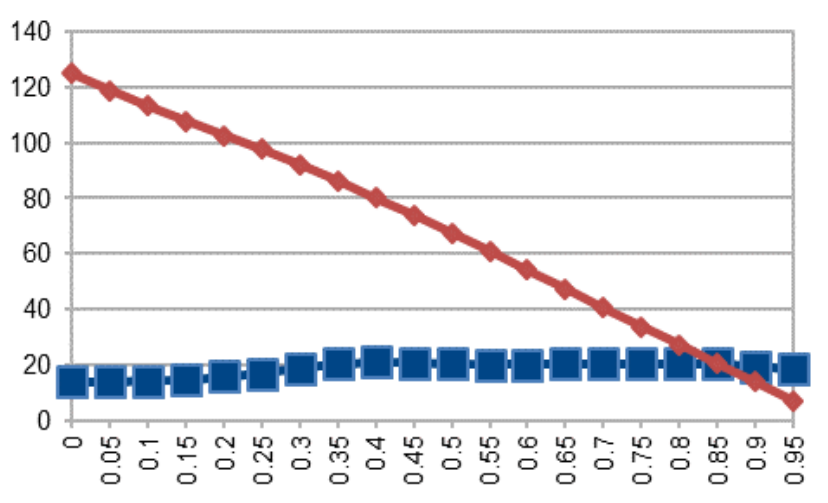

Fig. 15. Pruning-accuracy/size graph for SVHN.

The first column in the tables II-XII specifies the desired reduction in the size of the input dataset. The first row represents the SVM model trained without any pruning. The second column ("ACC") represents the accuracy of the trained SVM model. The third column ("NZV") presents the number of non-zero attributes in the support vectors, while the fourth ("ZV") contains the number of zero attributes introduced during the pruning process.

The fifth column ("Reduction") presents the relative size of the trained sparse SVM model compared to the original dense SVM model size shown in the first row of each table. This column shows how effective the pruning of SVM modules is when the AST-SVM algorithm. In an ideal case, the value of this column should be equal to 1 - "Target" value specified in the first column, but this is rarely the case. The reason for this is that when the input dataset is pruned, more support vectors are usually needed to achieve better accuracy as it was described in Chapter II. As Tables II-XII 
indicate, the reduction in the size of the trained SVM module is almost always possible. Please notice, when the reduction in trained SVM size is possible, it is always less than the specified target reduction value.

For some of the datasets, the reduction of the size of the trained SVM model can even help to achieve better classification accuracy. This is the case with the SVHN dataset, which is the biggest dataset in the experiments. In this case, the sparse SVM model can be almost 2 times smaller and achieve accuracy, which is over $7 \%$ better than the accuracy of the dense SVM model. One explanation for this unexpected behavior could be that the input dataset contains a lot of noise in the data, which pruning helps to eliminate.

The sixth column shows the speed-up that is possible to achieve when using ASA-SVM architecture, compared to RMLC architecture, to implement the SVM model. With the reduction of trained SVM model size, fewer operations are needed to classify input instance. Every multiplication with the zero-valued support vector attribute can be skipped in the ASA-SVM accelerator, resulting in better performance and better energy usage. Please notice, that for some datasets, the original model also contains some zeros in its support vectors. In these cases, even without the pruning ASA-SVM architecture would enable speed-up over previously proposed RMLC architecture. From the Tables II-XII, it can be concluded that the speed-up between 1.17 and 7.92 is possible, taking into account the accepted classification accuracy reduction of $2 \%$ during the SVM pruning process.

The seventh column shows achievable relative memory reduction, when only the NZVs of pruned SVM model are stored, together with their increment values, compared to memory size required to store the dense SVM model. Values greater than one indicate that memory usage is being increased, while values smaller than one represent a situation when memory footprint is decreased. In case when dense SVM model doesn't contain the significant number of zero-valued support vector attributes, and the SVM pruning percentage is close to zero percent, the memory size required to store SVM model parameters will increase. This is because every NZV value has to be stored together with its corresponding increment value. In this case, a significant number of support vector attribute values will be different from zero, and the increments will be just additional information needed to be stored without any benefit to speeding up the instance classification process. However, when the SVM model can be pruned with higher pruning rates, a significant reduction in memory will be possible. With the accepted trained SVM accuracy drop of $2 \%$ compared to the dense SVM model, it can be concluded from the data shown in Tables II-XII that the SVM size memory reduction from $3 \%$ up to $85 \%$ is achievable.

\section{Conclusions}

This paper proposes a novel algorithm for training of sparse SVM models, called AST-SVM, and a hardware architecture for the acceleration of sparse SVM models, called ASA-SVM, which can take advantage of sparse SVMs and achieve better performance compared to the previously proposed hardware architectures that accelerate only dense SVMs.

Performed experiments, using 20 standard datasets, clearly indicate that using sparse over dense SVMs has two advantages, the memory size required to store the SVM model is reduced, and the input instances are processed faster.

When created SVM model size is considered, a reduction from $3 \%$ to $85 \%$ is possible, when using sparse instead of dense SVMs.

Additionally, the experiments clearly show that the sparse SVM models enable faster instance processing when using the hardware accelerator specifically designed to process sparse SVMs. Instance processing time speedup from 1.17 to 7.92 was reported on selected datasets.

\section{CONFLICTS OF INTEREST}

The authors declare that they have no conflicts of interest.

\section{REFERENCES}

[1] C. Cortes and V. Vapnik, "Support-vector networks", Mach. Learn vol. 20, pp. 273-297, 1995. DOI: 10.1007/BF00994018.

[2] X.-X. Niu and Ch. Y. Suen, "A novel hybrid CNN-SVM classifier for recognizing handwritten digits", Pattern Recogn., vol. 45, no. 4, pp. 1318-1325, Apr. 2012. DOI: 10.1016/j.patcog.2011.09.021.

[3] M. Elleuch, R. Maalej, and M. Kherallah, "A new design based-SVM of the CNN classifier architecture with dropout for offline Arabic handwritten recognition", Procedia Comput. Sci., vol. 80, pp. 1712 1723, Jun. 2016. DOI: 10.1016/j.procs.2016.05.512.

[4] N. Cristianini and J. Shawe-Taylor, An Introduction to Support Vector Machines and Other Kernel-based Learning Methods. Cambridge, Cambridge Univ. Press, 2000. DOI: 10.1017/CBO9780511801389.

[5] U. Kressel, "Pairwise classification and support vector machines", in Advances in Kernel Methods: Support Vector Learning. MIT Press, Cambridge, MA, USA, 1999, pp. 255-268.

[6] J. Platt, "Sequential minimal optimization: A fast algorithm for training support vector machines", Technical Report MSR-TR-98-14, Apr. 1998.

[7] R. Fan, P. Chen, and C. Lin, "Working set selection using second order information for training SVM", Journal of Machine Learning Research, vol. 6, pp. 1889-1918, 2005.

[8] W. Chen, J. Wilson, S. Tyree, K. Weinberger, and J. Chen, "Compressing neural networks with the hashing trick", in Proc. of International Conference on Machine Learning, vol. 37, 2015, pp. 2285-2294. arXiv: 1504.04788.

[9] S. Han, H. Mao, and W. J. Dally. "Deep compression: Compressing deep neural network with pruning, trained quantization and Huffman coding", in Proc. of 4th International Conference on Learning Representations, ICLR 2016, San Juan, Puerto Rico, May 2016. arXiv: 1510.00149.

[10] S. Han, J. Pool, J. Tran, and W. J. Dally, "Learning both weights and connections for efficient neural network", in in Proc. of NeurIPS 2015 Twenty-ninth Conference on Neural Information Processing Systems, 2015, pp. 1135-1143. arXiv:1506.02626.

[11] F. N. Iandola et al., "Squeezenet: Alexnet-level accuracy with 50x fewer parameters and $<0.5 \mathrm{mb}$ model size", in Proc. of 5th International Conference on Learning Representations, 2017. arXiv: 1602.07360 .

[12] S. S. Keerthi, O. Chapelle, and D. DeCoste, "Building support vector machines with reduced classifier complexity", Journal of Machine Learning Research, vol. 7, pp. 1493-1515, 2016.

[13] C. J. Burges and B. Scholkopf, "Improving the Accuracy and Speed of Support Vector Machines", in Advances in neural information processing systems 1997 pp. 375-381.

[14] D. Anguita, A. Boni, and S. Ridella, "A digital architecture for support vector machines: Theory, algorithm, and FPGA implementation", IEEE Transactions on Neural Networks, vol. 14, no. 5, pp. 993-1009, 2003. DOI: 10.1109/TNN.2003.816033.

[15] D. Anguita, L. Carlino, A. Ghio, and S. Ridella, "A FPGA core generator for embedded classification systems", Journal of Circuits, Systems, and Computers, vol. 20, no. 2, pp. 263-282, 2011. DOI: $10.1142 / \mathrm{S} 0218126611007244$.

[16] M. Davood, A. Soleimani, H. Khosravi, and M. Taghizadeh, "FPGA simulation of linear and nonlinear support vector machine", Journal 
of Software Engineering and Applications, vol. 4, no. 5, pp. 320-328, 2011. DOI: $10.4236 /$ jsea.2011.45036.

[17] M. Papadonikolakis and C. Bouganis, "Novel cascade FPGA accelerator for support vector machines classification", IEEE Transaction on Neural Networks Learning Systems, vol. 23, no. 7, pp. 1040-1052, 2012. DOI: 10.1109/TNNLS.2012.2196446.

[18] C. Kyrkou and T. Theocharides, "A parallel hardware architecture for real-time object detection with support vector machines", IEEE Transactions on Computers, vol. 61, no. 6, pp. 831-842, 2012. DOI: 10.1109/TC.2011.113.

[19] V. Vranković and R. Struharik, "New architecture for SVM classifier and its application to telecommunication problems", in Proc of. 19th Telecommunications Forum (TELFOR), Belgrade, 2011, pp. 1543-
1545. DOI: 10.1109/TELFOR.2011.6143852.

[20] V. Vranjkovic, R. Struharik, and L. Novak, "Reconfigurable hardware for machine learning applications", Journal of Circuits, Systems, and Computers, vol. 24, no. 5, 2015. DOI: 10.1142/S0218126615500644.

[21] "Xilinx Zynq UltraScale+ MPSoC ZCU102 Evaluation Kit", XILINX. [Online]. Available: https://www.xilinx.com/products/boards-and-kits/ek-u1-zcu102g.html

[22] "LIBSVM - A Library for Support Vector Machines". [Online]. Available: https://www.csie.ntu.edu.tw/ cjlin/libsvm/

[23] "LIBSVM Data: Classification, Regression, and Multi-label". [Online].

https://www.csie.ntu.edu.tw/ cjlin/libsvmtools/datasets/ 